Supporting Information

\title{
Natural Leukocyte Membrane-Masked Microelectrodes with Enhanced Antifouling Ability and Biocompatibility for In Vivo Electrochemical Sensing
}

Huan Wei, ${ }^{\dagger} \S$ Fei Wu, ${ }^{*} \dagger, \S$ Lijuan Li, ${ }^{\star}$ Xiaoti Yang, ${ }^{\dagger, \S}$ Cong Xu ${ }^{\dagger, \S}$ Ping Yu, ${ }^{\dagger, \S}$ Furong Ma, ${ }^{\ddagger}$ Lanqun Mao ${ }^{*},, \S$

${ }^{\dagger}$ Beijing National Laboratory for Molecular Sciences, Key Laboratory of Analytical Chemistry for Living Biosystems, Institute of Chemistry, The Chinese Academy of Sciences, Beijing 100190, China.

* Department of Otorhinolaryngology, Peking University Third Hospital, Beijing 100083, China

$\S$ University of Chinese Academy of Sciences, Beijing 100049, China.

\section{Table of contents}

Figure S1. Schematics of the preparation process of LM and its construction.

Figure S2. Purification of leukocyte membrane through discontinuous density sucrose gradient.

Figure S3. amperometric responses and calibration curves of bare CFE and APTES-coated CFE towards DA in pure aCSF and homogenate-containing aCSF.

Figure S4. amperometric responses and calibration curves towards DA at bare CFE and APTES-coated CFE before and after in vivo implantation in the cortex of rat brain for $8 \mathrm{~h}$. 
*Corresponding Author. E-mail: 1qmao@iccas.ac.cn, wufei317@iccas.ac.cn.



Figure S1. Schematics of the preparation process of LM and its construction. 
A

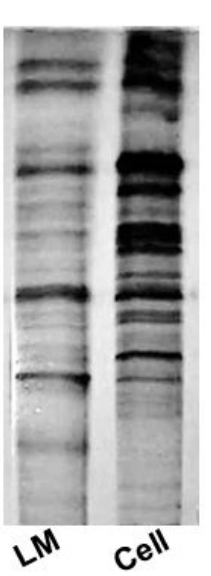

B

CD45

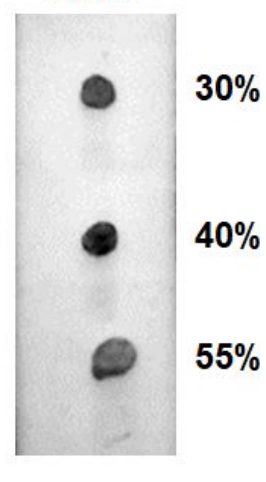

Figure S2. (A) SDS-PAGE analysis of membrane proteins in membrane fragments (MF) and whole cells. (B) Immunoblotting of membrane protein CD45 in different sucrose bands. 

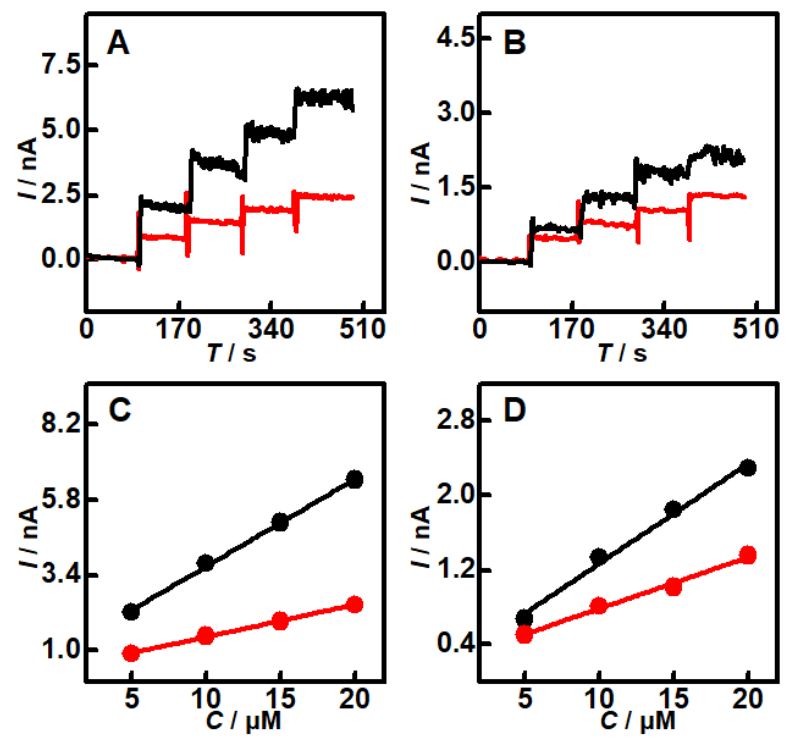

Figure S3. Typical amperometric responses of bare CFE (A) and APTES-coated CFE (B) towards successive additions of DA (each addition, $5 \mu \mathrm{M}$ ) in pure aCSF (black curves) and homogenate-containing aCSF (red curves). Calibration curves were obtained with bare CFE (C) and APTES-coated CFE (D) in pure aCSF (black curves) and homogenate-containing aCSF (red curves). 

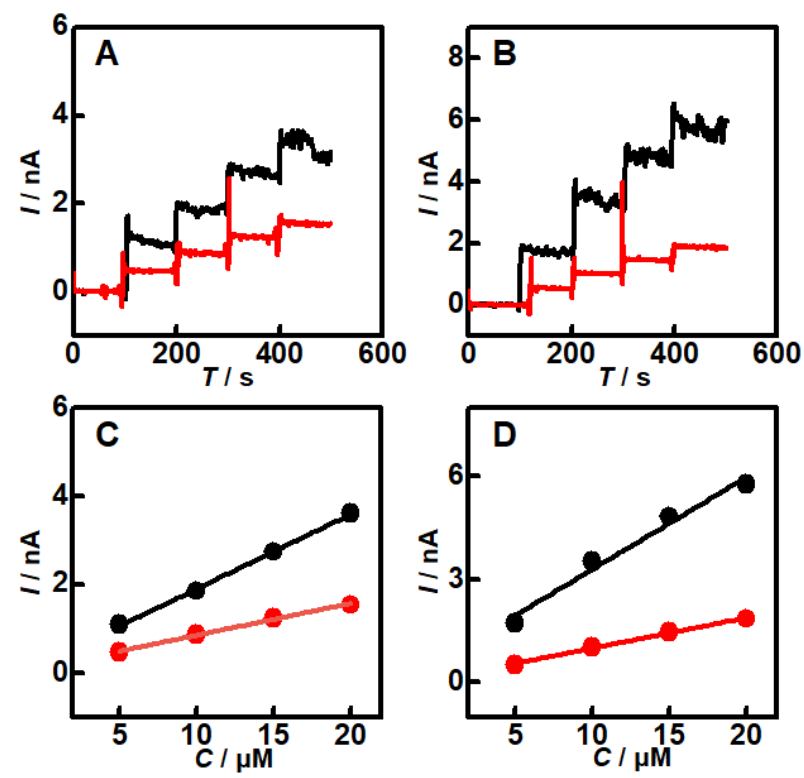

Figure S4. Typical amperometric responses of the APTES-coated CFE (A) and bare CFE (B) towards successive additions of DA in aCSF before (black curves) and after (red curves) in vivo implantation in the cortex of rat brain for $8 \mathrm{~h}$. Pre- (black curves) and post-calibration (red curves) were obtained with APTEScoated CFE (C) and bare CFE (D) under the same condition of A and B. 